\title{
El estudio experimental del castigo: una revisión**
}

\section{Manuel Alonso}

Universidad de Granada

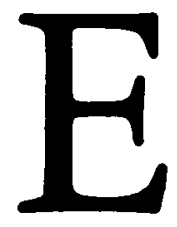

El objetivo de este trabajo es ofrecer una puesta al día de tres aspectos dentro del tema general del estudio experimental del castigo, fundamentalmente en organismos no humanos: simetría vs. asimimetría, contingencia y variables relevantes. En los dos primeros se realiza, además, un recorrido histórico.

La publicación en el año 1898 (Thorndike, 1898) de la tesis doctoral que Edward Lee Thorndike había presentado en la Columbia University, significa, primordialmente, una nueva aproximación a un viejo tema. La creencia, a nivel popular, de la efectividad de premios y castigos para cambiar conductas, era el viejo tema. La gran aportación de Thorndike, su nueva aproximación, fue hacer pasar estas ideas que flotaban en el ambiente a través del tamiz de la experimentación.

Los sujetos con los que realizó sus experimentos fueron gatos, perros y pollitos. Los estudios con gatos, que ejercieron gran influencia, comenzaban introduciendo uno hambriento por la parte superior de una caja. Una vez dentro, el animal

- El presente trabajo es la reformulación de una parte de la Memoria de Licenciatura realizada por el autor en la Universidad Complutense de Madrid, bajo la dirección del profesor Víctor García Hoz Rosales.

- Doy las gracias por los valiosos comentarios hechos a una primera versión de este trabajo a los profesores José Bernia y Pio Tudela; no obstante, cualquier deficiencia en la actual es únicamente imputable a su autor. 
debía acertar a manipular un sencillo mecanismo que hacía que una puerta lateral se abriera, dando posibilidad al animal a acceder a un poco de comida, que se encontraba a poca distancia de la caja. Terminado este ciclo, se introducía al animal de nuevo en la caja, iniciándose otro ciclo, y así sucesivamente. Thorndike, al anotar cuidadosamente la duración de cada ciclo, descubrió una correlación negativa entre el número de veces que el animal había logrado salir de la caja y el tiempo que tardaba en hacerlo cada vez; a mayor número de veces que el animal había logrado salir, correspondía un menor tiempo en la realización de un ciclo.

Fue trece años más tarde (Thorndike, 1911) cuando acuñó formalmente el término «ley del efecto», cuya formulación más sencilla nos dice que las consecuencias satisfactorias fortalecen la asociación entre estímulo y respuesta, y las consecuencias insatisfactorias la debilitan.

Dentro de la ley del efecto, es necesario distinguir entre la ley empírica del efecto y la ley teórica del efecto. La primera revela que las consecuencias contingentes a una respuesta, cambian la probabilidad de dicha respuesta. La ley teórica del efecto, surge al proponer Thorndike la explicación de por qué y cómo ocurre este fenómeno. Para él, que los animales aprendieran a salir cada vez más rápidamente de la caja, implicaba formarse una asociación entre el dispositivo-estímulo que permitía abrir la puerta, y la respuesta de manipularla con éxito. Esta conexión estaba influida por sus consecuencias contingentes, en la medida de que la satisfacción que el animal experimentaba alcanzando la comida, hacía que dicha conexión se fortaleciese. De la misma manera, otras conexiones E-R que no conducían a este fin, terminaban por desaparecer. Así pues, los cambios en probabilidad eran explicados mediante cambios en la fuerza de la asociación E-R. Esta distinción es de suma importancia puesto que, aparte de eliminar toda una serie de confusiones, cuando posteriormente se hable de una vuelta a este «primer» Thorndike, significa referirse a la ley empírica del efecto, en el sentido de que las consecuencias contingentes hacen que una respuesta ocurra más o menos frecuentemente en el futuro.

Como es fácilmente deducible, por estos años, para Thorndike, los «premios» y los castigos guardaban una estricta simetría: los primeros fortalecían una conexión; los segundos la debilitaban.

Al inicio de la década de los años treinta, la ley del efecto sufre una reformulación. Una serie de experimentos mostraron a Thorndike que las consecuencias contingentes a las respuestas no actuaban por igual, pero en sentido contrario, cuando estas consecuencias eran satisfactorias o insatisfactorias (Thorndike, 1931, 1932 a, b). Dos tipos principales de experimentos llevaron a Thorndike a esta conclusión. Uno de ellos fue sobre aprendizaje verbal. Se presentaba al sujeto una lista de palabras, teniendo cada una de ellas una serie de traducciones opcionales a otro idioma que el sujeto debía escoger. Thorndike afirmó que la recompensa (decirle al sujeto acorrecto» cuando daba con la opción verdadera), conducía al fortalecimiento de la conexión entre la palabra y la traducción escogida, mientras que el castigo, (decirle al sujeto «incorrecto» cuando la opción escogida no era la verdadera), no debilitaba dicha conexión. El otro tipo de experimento lo realizó con pollitos de trece a cincuenta días. En un laberinto se le presentaba a uno de ellos tres caminos; uno conducía à la compañía de otros pollitos que estaban comiendo; los otros dos a un encierro de treinta segundos en una caja pequeña. Los resulta- 
dos fueron evaluados por Thorndike en el sentido de que el «premio» (compañía y comida) siempre fortalecían la conexión sustancialmente y el castigo (encierro) la debilitaba poco o nada.

De esta manera, la ley simétrica del efecto quedó truncada al descartar Thorndike una de sus mitades, la que hacía referencia a la debilitación de las conexiones por sus consecuencias contingentes insatisfactorias.

\section{Asimetría vs. simetría}

Comenzamos retomando el tema a partir de la reformulación de la ley del efecto, dando cuenta de trabajos concordantes con este «segundo» Thorndike. Más adelante aportaremos una serie de autores cuya característica es el regreso al «primers Thorndike.

Skinner $(1938,1953)$ niega efectividad al castigo. Para él, ni el premio tiene efectos opuestos al castigo, ni éste reduce permanentemente las respuestas. Stone (1953), también aporta pruebas confirmatorias de la asimetría de la ley del efecto en un estudio efectuado con 160 sujetos. Sus resultados indicaron que decir «mal» a un sujeto fortalecía la respuesta en mayor medida que no decir nada.

El resultado de los experimentos de Estes (1944), apoyan también el punto de vista de la ley truncada del efecto. Concretamente dice:

"Los resultados de la presente investigación tienden, en general, a confirmar la más reciente formulación de la ley del efecto de Thorndike (Thorndike, 1935). Se muestra que el efecto del castigo en la respuesta castigada es debido a una competición de reacciones creadas por el estímulo nocivo. No ha aparecido evidencia indicadora de que el castigo ejerza un efecto debilitante directo sobre una respuesta, comparable al fortalecimiento producido por el premio. (Estes, 1944; apartado VI: Implicaciones teóricas y prácticas).

Para finalizar el grupo de algunos investigadores que no aceptan la simetría de la ley del efecto, podemos citar a Bolles (1967), el cual apoya explícitamente el punto de vista del «segundo» Thorndike, afirmando que el castigo ni es eficaz, ni tiene efectos directos sobre la conducta.

Veamos ahora quiénes son los autores que propugnan una simetría de los efectos del "premio» y el castigo en la conducta.

Azrin (1956), al observar que los efectos del tipo de castigo que él utilizó en este experimento eran similares a los obtenidos mediante reforzamiento, pero inversos en cuanto a la dirección del cambio, afirma que los estímulos aversivos actúan sobre la conducta en dirección opuesta a los reforzantes. Azrin y Holz (1966) resuelven de manera clara este problema. Para ellos, el castigo y la recompensa son procesos antagónicos; ambos afectan inicialmente a la conducta en general, pero, posteriormente, su efecto se vuelve específico a una respuesta particular. Su única diferencia estriba en la manera de cambiar la frecuencia de la conducta a la cual son contingentes.

Rachlin y Herrnstein (1969) aconsejan hacer lo que en años anteriores comenzó a tomar cuerpo y, más tarde, ha ido confirmándose. Este consejo no es otro que olvidarse de la reformulación de la ley del efecto de Thorndike (1931, 1932 a, b), 
y aceptar como válida, únicamente, su concepción original, es decir, Thorndike (1911). Ellos escriben:

\section{"... Los autores recomiendan un regreso a la primera formualción} de la ley del efecto de Thorndike, en la cual dos mecanismos conducen a efectos opuestos en la conducta». Rachlin y Herrnstein (1969, p. 108).

Tanto las pruebas experimentales como las concepciones teóricas acerca de la simetría de los efectos del reforzamiento y del castigo, continúan hasta nuestros días. Como nuevos puntos de referencia podemos tomar, junto a otros muchos, a Church (1969), Estes (1969), Fantino (1973), Farley y Fantino (1978), Morse y Kelleher (1977), Schuster y Rachlin (1968) y Terhune y Premack (1974).

\section{EL problema de la contingencia}

El castigo hace referencia a un estímulo aversivo presentado contingentemente a una respuesta, cuyo efecto es la reducción en la futura probabilidad de dicha respuesta; ahora bien, un estímulo aversivo no contingente también produce cierto grado de reducción. Un ejemplo de este último procedimiento sucede cuando un estímulo neutro, llamado preaversivo, precede invariablemente a un estímulo aversivo primario, se presenta sin relación con la respuesta y es inevitable por parte del animal. Si ambos se superponen a una serie de respuestas reforzadas positivamente, se aprecia una reducción en la tasa de dichas respuestas, fenómeno conocido como ansiedad, respuesta emocional condicionada (R.E.C.) o supresión condicionada. Estes y Skinner (1941), midieron los efectos supresores de este procedimiento en la conducta. Después de que un programa de reforzamiento mantuviera en una tasa estable las opresiones de una palanca por unas ratas, ocasionalmente se presentó un tono por cierto tiempo, cuyo final se hizo coincidir con un shock. Alrededor del cuarto día de administración, las respuestas prácticamente cesaron al iniciarse la presentación del tono. Este efecto no es debido a la presentación contingente del shock, puesto que éste se dio independientemente de la respuesta, sino, según los autores, a un estado emocional producido por el tono, el cual anticipó el estímulo aversivo.

En línea con este trabajo, y utilizando también la técnica de R.E.C., está la monografía de Estes (1944). En ella hay dos experimentos (B e I) que tienen relación con el problema que estamos tocando. En el experimento B se estudian, mediante cuatro grupos de ratas, los efectos de la extinción y del castigo. En el experimento I se analizan los efectos de un shock no correlacionado con la respuesta y de la extinción, utilizando dos grupos de cuatro ratas cada uno. De la comparación de ambos experimentos resultó que no había diferencia en ejecución entre el grupo que recibía castigo y el grupo que recibía shock no correlacionado con la respuesta, lo que le llevó a afirmar que:

"No hay evidencia de que la correlación del castigo con la respuesta per se, en el papel de un "reforzamiento negativo", sea importante». Estes (1944). (Sumario de principios teóricos, apartado II).

Resultados similares obtuvieron Hunt y Brady (1955). Estos autores hallaron casi completa supresión de la respuesta tanto en un grupo en el cual el shock era 


\section{Estudios}

contingente a la respuesta en presencia de un ruido (grupo castigado), como en otro grupo en el cual el shock seguía al ruido y era no contingente con la respuesta (grp. R.E.C.).

Con Azrin (1956), comienzan una serie de experimentos que demuestran la superioridad del procedimiento del castigo sobre el de R.E.C. en cuanto a su eficacia para suprimir las respuestas, o dicho de otra manera, la superioridad del procedimiento del shock contigente sobre un método de reducción de respuestas que utiliza un shock no contingente. Este autor encontró que el castigo era mucho más efectivo que la presentación de shock no contingente para reducir las respuestas dadas en presencia de un estímulo preaversivo, independientemente de que el estímulo aversivo se presentase a intervalos fijos o variables. El factor decisivo resultó ser la contingencia entre la respuesta y el estímulo aversivo.

Schuster y Rachlin (1968) entrenaron a pichones para que respondieran en dos diferentes condiciones, ambas mantenidas por idéntico programa de reforzamiento positivo de intervalo variable. Durante una condición, cada respuesta fue castigada con shock; durante la otra, el shock fue presentado de manera no contingente a la respuesta. Los resultados mostraron que el castigo causó mayor supresión que el shock independiente, incluso cuando la tasa del shock independiente fue mucho mayor. Rachlin y Herrnstein (1969), aun llegando a idénticos resultados, hacen importantes matizaciones:

1. "La respuesta es escasamente suprimida por un shock no contingente, excepto en altos niveles de intensidad o razón de presentación."

2. "El shock contingente suprime la respuesta más que el shock no contingente de igual intensidad, incluso cuando el sbock no contingente es más frecuente." (Pág. 98).

Frankel (1975) obtiene mayor supresión de la respuesta con el procedimiento contingente que cuando empleó shock no contingente, en dos experimentos. En el experimento 1, estos resultados fueron estables durante ocho días de administración de shock; en el experimento 2, se compararon tres niveles bajos de shock y las diferencias nuevamente se mantuvieron.

A similares conclusiones han llegado también otros autores (Boe y Church, 1967; Church, 1963, 1969; Mackintosh, 1974; Rachlin, 1976; Schwartz, 1978; Tarpy, 1975).

La disparidad de toda esta serie de resultados con los de Estes (1944) y Hunt y Brody (1955) pudiera haber sido debida al hecho de que en estos dos trabajos la administración del castigo se limitó a un período total inferior a una hora y a una única sesión.

\section{Variables relevantes}

Las variables más importantes que afectan a la efectividad del castigo las hemos clasificado en dos grupos. El primero se refiere a cómo presentemos el estímulo aversivo; el segundo a las características de la conducta castigada. Unicamente se da cuenta de ellas telegráficamente, debiendo acudir a la/s referencia/s particular/es cuando se desee una información más amplia. 


\section{En cuanto al estimulo}

a) Modo de administración

Una elẹvada intensidad de estimulación aversiva presentada de súbito, puede conseguir una completa supresión de la respuesta; esta misma intensidad, o incluso otra mayor, lograda gradualmente desde niveles inferiores, acarreará únicamente una supresión parcial (Azrin y Holz, 1966; Azrin, Holz y Hake, 1963; Fantino, 1973; Miller, 1960).

b) Intensidad

Cuanto mayor sea la intensidad del castigo, más grande será la reducción de la respuesta castigada, hasta llegar a un punto en que la supresión será completa (Appel, 1963; Appel y Peterson, 1965; Azrin, 1960; Hake, Azrin y Oxford, 1967; Powell, 1970).

\section{c) Duración}

Una gran cantidad de experimentos ofrecen evidencia de que la duración de la estimulación aversiva actúa sobre la respuesta castigada de manera similar a como lo hace la intensidad (Appel, 1968). Esto nos induce a poder afirmar que a mayor duración del estímulo aversivo, mayor supresión de la respuesta se logrará (Church, Raymond y Beauchamp, 1967).

\section{d) Contigüidad}

Los resultados ofrecidos por gran cantidad de experimentos son concluyentes: la estimulación aversiva presentada inmediatamente después de la respuesta, acarrea mayor supresión que otra demorada (Baron, 1965; Camp, Raymond y Church, 1967; Cohen, 1968; Church, 1963, 1969).

\section{e) Programas}

En igualdad de condiciones, el castigo continuo ejerce mayor supresión que el castigo intermitente, pero la respuesta castigada se reestablece más rápidamente después del castigo continuo (Azrin, Holz y Hake, 1963; Estes, 1944, experimentos E. y K.; Filby y Appel, 1966).

Aun cuando en el castigo de respuestas bajo reforzamiento negativo aparecen algunos fenómenos particulares (ver Brown, 1969 y crítica de Bolles, 1975), las variables hasta ahora tratadas tienen similares efectos. En lo relativo al modo de presentación, un shock presentado a una particular intensidad, acarrea mayor supresión que otro que logra la misma intensidad desde niveles inferiores (Powell y Morris, 1969). En cuanto a la intensidad, a mayor intensidad, mayor decremento de la tasa de respuesta (Powell y Morris, 1969; Seligman y Campbell, 1965; Smith, Misanin y Campbell, 1966). La duración del castigo también tiene similares efectos en conductas bajo reforzamiento negativo que bajo reforzamiento positivo: a mayor duración del castigo, le correspondió mayor supresión de la conducta de evitación (Misanin y col., 1966; Smith y col., 1966; Seligman y Campbell, 1965). Por último, un aumento de la demora disminuyó los efectos supresores del castigo (Kamin, 1959; Misanin y col., 1966). 


\section{Estudios}

Aunque sólo sea mínimamente, es interesante dar cuenta, dentro de este apartado, de los resultados de un experimento realizado por Hoz y Azrin (Holz y Azrin, 1961), el cual ha tenido una gran transcendencia. En síntesis, estos investigadores hallaron que la presentación de un shock contigente severo podía llegar a aumentar la tasa de respuesta y que la presentación de un shock ligero contingente (20 volt.) podía reducirla drásticamente, como resultado de haberse convertido el shock en un $E^{D}$ de reforzamiento y extinción respectivamente.

\section{En cuanto a la respuesta}

\section{a) Programas de reforzamiento}

Las consecuencias de castigar respuestas mantenidas por programas simples $R V$ y RF fueron similares a las observadas bajo programas múltiples RV-RF (Powell, 1970). Cuando la intensidad del shock se incrementa, las respuestas bajo un programa de reforzamiento RV acusan más los efectos del castigo que las respuestas bajo un programa de reforzamiento IV (Powell, 1970). Ver también Azrin y Holz, 1966; y Fantino, 1973).

b) Privación

El grado de privación de un sujeto cuya conducta está mantenida por reforzamiento alimenticio, es un poderoso determinante de los efectos del castigo que apliquemos a dicha conducta. En general, a mayor privación, corresponde un menor efecto del castigo (Azrin, 1960; Azrin, Holz y Hake, 1963).

\section{c) Respuesta alternativa}

Si disponemos de dos sujetos, uno con la posibilidad de dar una única respuesta y el otro pudiendo ejecutar dos, y castigamos con la misma intensidad una respuesta de cada uno de ellos, el castigo será más efectivo - reducirá en mayor medida la tasa de respuesta - en el caso del sujeto con respuesta alternativa (Herman y Hazrin, 1964).

d) Experiencia de estimulación aversiva

La experiencia con castigo ligero reduce los efectos de un subsecuente castigo de mayor intensidad, mientras que la experiencia con un castigo fuerte incrementa los efectos de un posterior castigo de menor intensidad, denominándose este efecto ainercia conductual» (Cohen, 1968; Church, 1969; Hake, Azrin y Oxford, 1967; Karsch, 1963).

\section{Resumen}

El presente trabajo trata desde un punto de vista histórico, algunos de los aspectos del castigo. Señala como, actualmente, a la luz de numerosos experimentos, se aceptan como válidas las dos caras de la ley simétrica del efecto (ley positiva del efecto y ley negativa del efecto). Simetría que Thorndike, su formulador, desechó en los inicios de la década de los años treinta. También se ofrece evidencia de la capital importancia de la contingencia 
dentro del procedimiento de castigo (superioridad del castigo frente a R.E.C.). Finalmente se da cuenta de una serie de parámetros que ejercen una gran influencia en los efectos del castigo

\section{Summary}

From a histovical point of view, the present work deals with some aspects of punishment. It shows how, nowadays, in the light of numerous experiments, both sides of the symmetric law of effect are accepted as being valid (the positive law of effect and the negative law of effect). Symmetry, which Thorndike, its creator, rejected at the beginning of the thirties' decade. Evidence of capital importance in also offered of contingence in the procedure of punishment (superiority of punishment aginst R.E.C.). Finally one notices a series of parameters which exercise a great influence over the effects of the punishment.

\section{Resumé}

Cette étude traite, d'un point de vue historique, certains aspects de la punition. Elle met en évidence comment, actuellement, à l'issue de nombreuses expériences, son considérées comme valables les deux facettes de la loi symétrique de l'effet (loi positive de l'effet et loi négative de l'effet). Symétrie que Thorndike, son théoricien, a rejetée dans les débuts des années trente. On y fait ressortir également l'importance capitale de la contingenge á l'intérieur du processus de la punition (supériorité de la punition sur le R.E.C.). On y cite finalement una série de paramètres qui exercent une grande influence sur les effects de la punition.

\section{Referencias}

APPEL, J. B.: Punishment and shock intensity. Science, 1963, 141, 528.529.

APPEL, J. B.: Fixed-interval punishment. Joumal of the Experimental Analysis of Behavior, 1968, 11, 803-808.

APPEL, J. B. y PETERSON, N. J.: Punishment: Effects of shock intensity on response suppression. Psychological Reports, 1965, 16, $721-730$.

AZRIN, N. H.: Some effects of two intermittent schedules of inmediate and non-inmediate punishment. Jourmal of Psychology, 1956, 42, 3-21.

AZRIN, N. H.: Effects of punishment intensity during variable-interval reinforcement. Joumal of the Experimental Analysis of Behavior, 1960, 3, 123-142.

AZRIN, N. H. y HOLZ, W. C.: Punishment. En W. K. Honing (Ed.). Operant Behavior: areas of research and application. Nueva York: Appleton-Century-Crofts, 1966 (trad. cas., Trillas, 1975).

AZRIN, N. H., HOLZ, W. C. y HAKE, D.: Fixet-ratio punishment. Journal of the Expermental Analysis of Behavior, 1963, 6, 141-148.

BARON, A.: Delayed punishment of a runway response. Joumal of Comparative and Physiological Psichology, 1965, 60, 131-134.

BOE, E. E., y CHURCH, R. M.: Permanent effects of punishment during extinctin. Joumal of Comparative and Pshysiological Psichology, 1967, 63, 486-492.

BOLLES, R. C.: Theory of motivation. Nueva York: Harper \& Row, 1967 (Trad. cas. Trillas, 1973).

BOLLES, R. C.: Theory of motivation (2. ${ }^{2}$ ed.) Nueva York: Harper \& Row, 1975.

BROWN, J. S.: Factors affecting self-punitive locomotor behavior. En B. A. Campbell y R. M. Church (ed.), Punishment and Aversive Behavior. Nueva York: Appletori-Century-Crofts, 1969.

CAMP, D. S., RAYMOND, G. A., y CHURCH, R. M.: The remporal relationship between response and punishment. Joumal of the Experimental Psychology, 1967, 74, 114-123.

COHEN, P. S.: Punishment: The interactive effects of delay and intensity of shock. Journal of the Experimental Analysis of Behavior, 1968, 11, 789-799.

CHURCH, R. M.: The varied effects of punishment on behavior. Psychological Review, 1963, 70, 369-402.

CHURCH, R. M.: Response suppression. En B. A. Campbell y R. M. Church (eds.) Punishment and aversive behavior. Nueva York: Appleton-Century-Crofts, 1969.

CHURCH, R. M., RAYMOND, G. A: y BEAUCHAMP, R. D.: Response suppression as a function of intensity and duration of a punishment. Journal of Comparative and Physiological Psychology, 1967, 63, 39-44. 
ESTES, W. K.: An experimental study of punishment. Psychological Monographs, 1944, 57 (M. ${ }^{\circ} 236$ comp.).

ESTES, W. K.: Outline of a theory of punishment. En A. B. Campbell y R. M. Church (eds.), Punishment and aversive behavior. Nueva York: Appleton-Century-Crofts, 1969.

ESTES, W. K. y SKINNER, B. F.: Some quantitative properties of anxiety. Joumal of Experimental Psychology, $1941,29,390-400$.

FANTINO, E.: Averssive control. En J. A. Nevin y G. S. Reynolds (eds.), The study of behavior. Glenview, Illinois: Scott, Foresman, 1973.

FARLEY, J. y FANTINO, E.: The symmetrical law of effect and the matching relation in choice behavior. Journal of the Experimental Analysis of Behavior, 1978, 29, 37-70.

FILBY, Y. y APPEL, J. B.: Variable-interval punishment during variable-interval reinforcement. Joumal of the Experimental Analysis of Behavior, 1966, 9, 521-527.

FRANKEL, F. D.: The role of response-punishment contingency in the suppression of positively-reinforced operant. Leaming ad Motivation, 1975, 6, 385-403.

HAKE, D. F., AZRIN, N. H. y OXFORD, R.: The effects of punishment intensity on squirrel monkeys. Joumal of the Experimental Analysis of Behavior, -1967, 10, 95.107.

HERMAN, R. L. y AZRIN, N. H.: Punishment by noise in a alternative response situation. Joumal of the Experimental analysis of Behavior, 1964, 7, 185-188.

HOLZ, W. C. y AZRIN, N. H.: Discriminative properties of punishment. Journal of the Experimental Analysis of Behavior, 1961, 4, 225-232.

HUNT, H. F. y BRADY, J. V.: Some effects of punishment and intercurtent eanxiety» on a simple operant. Joumal of Comparative and Physiological Psychology, 1955, 48, 305-310.

KAMIN, L. J.: The delay-of-punishment gradient. Joumal of Comparative and Physiological Psichology, 1959, $52,434.437$

KARSH, E. B.: Changes in intensity of punishment: Effects on running behavior of rats. Science, 1963, 140 , $1084-1085$

MACKINTOSH, N. J.: The psychology of Animal Learning. Nueva York: Academic Press, 1974.

MILLER, N. E.: Learning resistance to pain and fear; effects of overlearning, exposure and rewarded exposure in context. Journal of Experimental Psychology, 1960, 60, 137-145.

MISANIN, J. R., CAMPBEL, B. A. y SMITH, N. F.: Duration of punishment and the delay of punishment gradient. Canadian Joumal of Psychology, 1966, 20, 407-412.

MORSE, W. H. y KELLEHER, R. T.: Determinant of reinforcement and punishment. En W. K. Honig y J. E. R. Staddon (eds.), Handbook of operant bebavior. Englewood Cliffs, Nueva Jersey: PrenticeHall, 1977.

POWELL, R. W.: The effects of punishment shock intensity upon responding under multiple schedules. Journal of the Experimental Analysis of Behavior, 1970, 14, 201-211.

POWELI, R. W. y MORRIS, G.: Continuous punishment of free-operant avoidance in the rat. Joumal of the Experimental Analysis of Behavior, 1969, 12, 149-157.

RACHLIN, H.: Introduction to modern behaviorism (2. ${ }^{2}$ ed.). San Francisco: W. H. Freeman ad Company, 1976 (trad. cast. Debate, 1977).

RACHLIN, H. y HERRNSTEIN, R. J.: Hedonism revisited: On the negative law of effect. En B. A. Campbell y R. M. Curch (eds.), Punishment and aversive behavior. Nueva York: Appleton-Century-Crofts, 1969.

SCHUSTER, R. y RACHLIN, H.: Indifference between punishment and free shock: evidence for the negative law of effect. Joumal of the Experimental Analysis of Behavior, 1968, 11, 777-786.

SCHWARZT, B.: Psychology of Leaming and Behavior. Nueva York: Norton and Company, 1978.

SELIGMAN, M. E. P. y CAMPBELL, B. A.: Effect of intensity and duration of punishment on extinction of an avoidance response. Joumal of Comparative and Physiological Psychology, 1965, 59, 295-297.

SKINNER, B. F.: The behavior of organisms: an experimental analysis. Nueva York: Appleton-Century-Crofts, 1938, (trad. cas. Fontanella, 1975).

SKINNER, B. F.: Science and human bebavior. Nueva York: Macmillan, 1953. (trad. cas. Fontanella, 1970).

SMITH, M. C., MISANIN, J. R. y CAMPBELL, B. A.: Effect of punishment on extinction of an avoidance response: Facilitation or inhibition? Psychonomic Science, 1966, 4, 271-272.

STONE, G. R.: The effect of negative incentive in serial learning: VII. Theory of punishment. Journal of General Psychology, 1953, 48, 133-161.

TARPY, R. M.: Basic Principles of Learning. Glenview, Illinois: Scott, Foresman and Company, 1975. (trad. cas. Debate, 1977).

TERTHUNE, J. G. y PREMACK, D.: Comparison of reinforcement and punishment functions produced by some contigent event in the some subjets. Learning and Motivation, 1974, 5, 221-230.

THORNDIKE, E. L. : Animal intelligence. An Experimental study of the associative processes in animals. Psychological Monographs, $1898,2, \mathrm{n} .^{\circ} 8$ completo.

THORNDIKE, E. L.: Animal intelligence: Experimental studies. Nueva York Macmillan, 1911.

THORNDIKE, E. L.: Human learning. Nueva York: Appleton-Century-Crofts, 1931.

THORNDIKE, E. L.: The fundamentals of Leaming. Nueva York: Teachers College, Columbia University, 1932 (a).

THORNDIKE, E. L.: Reward and punishment in animal learning. Comparative Psychology Monographs, 1932,8 . (b).

THORNDIKE, E. L.: The psychology of want, interests and attitudes. Nueva York: Appleton-CenturyCrofts, 1935. 